\title{
BMJ Open Timeliness of diagnosis of breast and cervical cancers and associated factors in low-income and middle-income countries: a scoping review protocol
}

\author{
Chukwudi A Nnaji (D) , ${ }^{1,2}$ Paul Kuodi (D) , ${ }^{3}$ Fiona M Walter (D) , ${ }^{4}$ \\ Jennifer Moodley
}

To cite: Nnaji CA, Kuodi $P$, Walter FM, et al. Timeliness of diagnosis of breast and cervical cancers and associated factors in low-income and middleincome countries: a scoping review protocol. BMJ Open 2021;11:e044093. doi:10.1136/ bmjopen-2020-044093

- Prepublication history and additional supplemental material for this paper are available online. To view these files, please visit the journal online (http://dx.doi.org/10.1136/ bmjopen-2020-044093).

Received 24 August 2020 Revised 09 March 2021 Accepted 20 April 2021
Check for updates

(C) Author(s) (or their employer(s)) 2021. Re-use permitted under CC BY-NC. No commercial re-use. See rights and permissions. Published by BMJ.

For numbered affiliations see end of article.

Correspondence to Dr Chukwudi A Nnaji; nnjchu001@myuct.ac.za

\section{ABSTRACT}

Introduction Breast and cervical cancer are leading causes of morbidity and mortality in women globally, with disproportionately high burdens in low-income and middle-income countries (LMICs). While the incidence of both cancers increases across LMICs, many cases continue to go undiagnosed or diagnosed late. The aim of this review is to comprehensively map the current evidence on the time to breast or cervical cancer diagnosis and its associated factors in LMICs.

Methods and analysis This scoping review (ScR) will be informed by Arksey and O'Malley's enhanced ScR methodology framework. It will be reported in accordance with the Preferred Reporting Items for Systematic reviews and Meta-Analyses extension for Scoping Reviews. We will conduct a comprehensive search of the following electronic databases: MEDLINE (via PubMed), Cochrane Library, Scopus and the Cumulative Index to Nursing and Allied Health Literature (CINAHL). Two reviewers will independently screen all abstracts and full texts using predefined inclusion criteria. All publications describing the time to diagnosis and its associated factors in the contexts of breast or cervical cancer will be considered for inclusion. Evidence will be narratively synthesised and analysed using a predefined conceptual framework. Ethics and dissemination As this is a ScR of publicly available data, with no primary data collection, it will not require ethical approval. Findings will be disseminated widely through a peer-reviewed publication and forums such as conferences and community engagement sessions. This review will provide a user-friendly evidence summary for understanding the enormity of diagnostic delays and associated factors for breast and cervical cancers in LMICs, while helping to inform policy actions and implementation of interventions for addressing such delays.

\section{INTRODUCTION}

Breast and cervical cancer are leading causes of morbidity and mortality in women globally. ${ }^{2}$ The incidence of both cancers and their associated morbidity and mortality are disproportionately high in low-income and middle-income countries (LMICs). ${ }^{23}$ Breast cancer is the most common cancer among
Strengths and limitations of this study

- This scoping review will be conducted in accordance with an enhanced evidence synthesis methodology and will be reported using standard reporting guidelines.

- This review will update and fill an existing gap in the evidence relating to the nature of the time to diagnosis of breast and cervical cancer and associated factors associated in low-income and middle-income countries.

- Literature searches will be comprehensive, covering both peer-reviewed and relevant grey literature, with no language restrictions.

- Due to the broad nature of the topic, it is possible that some relevant literature may not be identified by our search strategy, however comprehensive.

women worldwide, with an age-standardised incidence rate (ASIR) of 31 per 100000 women in LMICs. ${ }^{45}$ Cervical cancer accounts for $16 \%$ of the cancer burden in LMICs, with an ASIR of 16 per 100000 women. ${ }^{2}$ Moreover, the number of new cases and subsequent cancer deaths in LMICs are expected to grow substantially in the coming decades. ${ }^{6}$ This growth has been attributed to population growth and increasing exposure to behavioural, dietary and environmental risk factors; in keeping with the epidemiological transition from communicable diseases to non-communicable diseases in developing countries. $^{16}$

Cancer mortality and survival are influenced by the timeliness of diagnosis and access to effective treatment. ${ }^{7-9}$ If diagnosed early, breast and cervical cancers are amenable to treatment with curative intent. ${ }^{10} 11$ Timely diagnosis is critical for optimising patients' navigation of the pathway from cancer symptom awareness to treatment. ${ }^{12-14}$ While the incidence of cancers increases across 
LMICs, many cases continue to go undiagnosed or diagnosed at a late stage when treatment is often less effective and more expensive. ${ }^{7815}$

In LMICs, timely cancer diagnosis is often constrained by patient and disease factors, as well as health system barriers. ${ }^{16-18}$ Patient factors include those that are demographic, psychological and behavioural, in addition to those associated with underlying sociocultural barriers to early cancer diagnosis such as lay beliefs. ${ }^{16} 1920$ Lay beliefs, such as perceptions that cancers are divine punishments for sin committed, are not uncommon in some communities in LMICs, as are views that cancers are contagious and that they are inevitably fatal. ${ }^{21}{ }^{22}$ Likewise, there are concerns that cancer treatment or surgery (such as mastectomy) may result in deformity and consequent divorce or family abandonment. ${ }^{1723}$ Furthermore, women in LMICs often fail to recognise or misinterpret cancer symptoms or wait until symptoms worsen before they seek medical attention. ${ }^{24}$ For instance women usually attribute the initial symptoms of cervical cancer to normal bodily changes or common illnesses such as sexually transmitted diseases. ${ }^{25}$

Disease factors include those related to the site, size, clinical manifestation and growth of tumours. ${ }^{18}$ Health system factors in LMICs include access, policy and service delivery barriers. Access barriers may be due to physical or geographical inaccessibility to health facilities and those associated with healthcare costs and affordability. ${ }^{16} 1820$ Service delivery barriers comprise factors such as inadequate diagnostic tools, poor referral systems and insufficient human resources. ${ }^{6} 1626$ Cancer diagnostic services in LMICs are often dependent on limited government funding, not consistently available or affordable, inequitably distributed and nested in fragmented health systems with complicated patient referral. ${ }^{1620}$

As a result of these, women in LMICs may be reluctant to seek care following their awareness of possible cancer symptoms, leading to delays in diagnosis. Consequently, a high proportion of patients are diagnosed at advanced stage, when treatment is often less effective and more expensive. ${ }^{16}{ }^{19}$ However, little is known about the extent to which these factors influence time to diagnosis, particularly in LMICs.

The measurement of time to cancer diagnosis is also complex. Complicating factors include those related to the patients, the clinical manifestation of cancers and the validity of diagnostic tools. ${ }^{27}$ Various methodological approaches for assessing time of diagnosis and diagnostic intervals exist, while their use in LMICs has grown over the years. ${ }^{22} 2628$ However, only a minority of these tools are theoretically informed and validated. ${ }^{27}$ Studies assessing appraisal intervals (time taken to interpret bodily changes/symptoms) and help-seeking intervals (time taken to act on those interpretations and seek help) commonly use tools that ignore existing models of patient behaviour, and are poorly or inadequately validated. ${ }^{18} 29$ To help address these measurement gaps, the distinct phases of cancer patients' pathways from symptom awareness to diagnosis and treatment have been conceptualised by the Model of Pathways to Treatment proposed by Walter, Scott and colleagues. ${ }^{18} 29$ According to the framework, there are five key events in the pathway to care: detection of bodily changes; perceived reasons to discuss symptoms with a healthcare provider; first consultation with a healthcare provider; diagnosis and start of treatment. The framework also identifies four important intervals between these phases: the appraisal, helpseeking, diagnostic and pre-treatment intervals. These events and processes represent particular moments at which barriers may exist and delay patients' access to care before or after a cancer diagnosis. ${ }^{70}$ In response to the well-recognised need to develop valid tool for measuring time to cancer diagnosis, the Aarhus checklist has been proposed for guiding the design and reporting of early cancer diagnosis studies. ${ }^{30}$

\section{STUDY RATIONALE}

Understanding the time to breast or cervical cancer diagnosis and associated factor is important to guide interventions for addressing diagnostic delays in LMICs. ${ }^{11} 3132$ In 2017, the WHO published the WHO guide to cancer early diagnosis. ${ }^{33}$ It provides a standard framework for systematically addressing barriers that may impede timely cancer diagnosis and treatment, which are particularly prevalent in LMICs. Addressing these barriers requires a conceptually sound understanding of the extent of time to diagnosis and the nature of the factors associated with such barriers. $^{34}$

This review aims to provide an up to date and comprehensive synthesis of the evidence on the time to breast or cervical cancer diagnosis and its associated factors in LMICs. Due to the possible lead time and length bias of cancer screening programmes (including those of breast and cervical cancer), this review will focus on early diagnosis of symptomatic breast and cervical cancers. Its findings will contribute to better understanding of the nature and extent of diagnostic timeliness and delays in resource-limited settings. In addition, it seeks to provide a systematically organised evidence summary for health policy makers, cancer programme managers, oncologists and other cancer care providers for guiding policy and practice decision making. Finally, it will be useful for informing the design of further interventions and strategies for addressing breast and cervical cancer diagnostic delays in resource-limited settings, while identifying opportunities for future research.

\section{METHODS AND ANALYSIS}

\section{Conceptual framework}

This scoping review $(\mathrm{ScR})$ will use the Model of Pathways to Treatment framework proposed by Walter, Scott and colleagues ${ }^{18} 29$ to map the identified evidence on the time intervals for breast or cervical cancer diagnosis and associated factors. The framework (See figure 1) specifies 


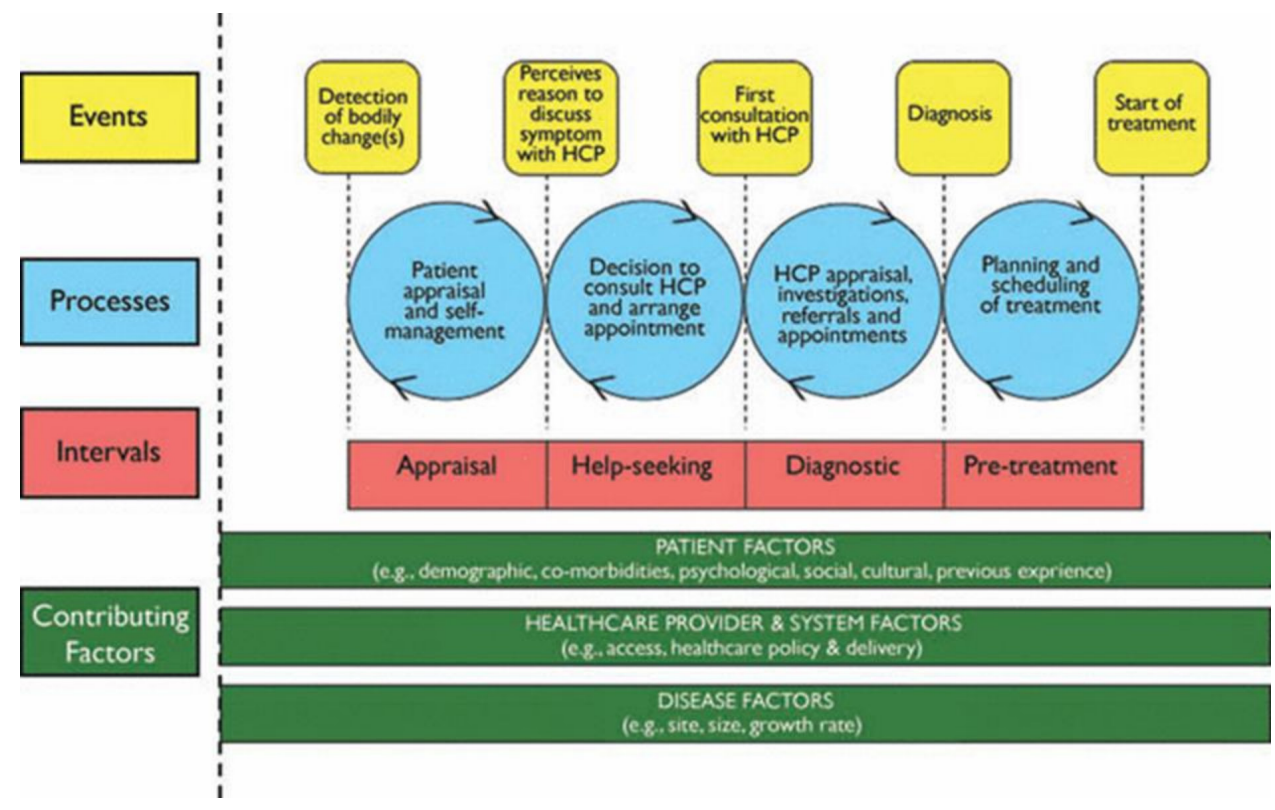

Figure 1 The model of pathways to treatment.

the essential events, processes and time intervals that may occur in the period prior to diagnosis and the start of medical treatment and identifies the factors that may contribute to the duration of each time interval.

\section{STUDY DESIGN}

This ScR is informed by Arksey and O'Malley's ScR methodology, ${ }^{35}$ as enhanced by Levac and colleagues. ${ }^{36}$ The enhanced framework involves six stages for undertaking a ScR: (1) identifying the research question; (2) identifying the relevant studies (defining the inclusion and exclusion criteria); (3) searching and selecting the evidence; (4) charting the evidence; (5) collating, summarising and reporting the evidence and (6) consultation with relevant stakeholders.

\section{STEP 1: IDENTIFYING THE RESEARCH QUESTION}

The ScR seeks to answer the questions: 'What is the current evidence on the time to breast and cervical cancer diagnosis and its associated factors in LMICs?'

Specifically, it will answer the following questions:

1. How is time to breast or cervical diagnosis defined or measured?

2. What are the time intervals for breast or cervical cancer diagnosis in LMICs?

3. What are the factors associated with time to diagnosis for breast and cervical cancers in LMICs?

\section{STEP 2: IDENTIFYING THE RELEVANT STUDIES (DEFINING THE INCLUSION AND EXCLUSION CRITERIA)}

In line with the research question, the inclusion criteria are defined using the using the population, concept and contexts (PCC) framework, proposed by Peters and colleagues. ${ }^{37}$ This framework is more appropriate for
ScRs than the population, intervention, comparator and outcome (PICO) framework commonly used for systematic reviews, as it allows for the consideration of publications that may not feature all of the four PICO elements (eg, lacking an outcome or comparator/control). Eligible population will include women with breast or cervical cancer and healthcare providers living in LMIC contexts. The concepts of interest for this review are time to diagnosis and diagnostic intervals for breast and/or cervical cancers. Time to diagnosis will include appraisal, help-seeking or diagnostic intervals as defined by model of pathways to treatment. ${ }^{1829}$

To be considered eligible for inclusion, studies will have to fulfil one or more of the following: (1) report-specific methods, tools or strategies for measuring time intervals in the context of breast and/or cervical cancer diagnoses in LMICs; (2) assess the time to diagnosis of breast and/or cervical cancers in LMIC settings; (3) evaluate the factors associated with diagnostic time intervals among women with breast and/or cervical cancers in LMICs. The definition of LMICs will be based on the World Bank's current classification using per capita gross national income. ${ }^{38}$ Multinational literature involving LMIC and non-LMIC countries and meeting inclusion criteria will be included, except where country-specific information cannot be abstracted. Similarly, articles involving multiple cancer types will be included, except where the relevant cancer type-specific information cannot be abstracted.

Articles focused solely or mainly on theoretical and conceptual understanding of timeliness of breast or cervical cancer diagnosis without assessing the timeliness of diagnosis in specific LMIC contexts will be excluded, as will those assessing cancer patient pathways that are not related to diagnostic time and intervals. Studies focused primarily on screening of asymptomatic individuals will also be excluded. Study design eligibility will include 
randomised trials, non-randomised trials and observational studies, with or without controls. However, inclusion will be limited to primary studies; while systematic, ScRs and other forms of aggregated evidence will be excluded.

\section{STEP 3: SEARCHING AND SELECTING THE EVIDENCE}

The literature search will be guided by the research questions and the PCC criteria. The search strategy will be developed with guidance from a health sciences subject librarian and will be applied in accordance with the Peer Review of Electronic Search Strategies guidelines. ${ }^{39}$ The search strategy will be pretested prior to the actual search. Search terms and free-text words will be combined using the Boolean operators 'AND' and 'OR', such as (breast OR cervical OR cervix, cancer OR neoplasm OR malignancy OR tumours) AND (diagnosis OR diagnostic OR detection OR discovery) AND (early OR timely OR time OR late OR delay). Search terms will include the use of controlled descriptors, such as Medical Subject Headings (MeSH) terms and Cumulative Index to Nursing and Allied Health Literature (CINAHL) headings, and their synonyms. In order to restrict search to LMICs, a location filter containing all countries currently classified as part of LMICs and synonymous geographical, regional and economic categorisations will be incorporated. See online supplemental appendix for provisional search strategy.

A comprehensive literature search will be conducted on the following electronic databases: MEDLINE (via PubMed), Cochrane Library (including the Cochrane Central Register of Controlled Trials (CENTRAL) and the Database of Abstracts of Reviews of Effects), Scopus, CINAHL and the International Clinical Trials Registry Platform. Additionally, relevant grey literature will be searched for potentially eligible articles, including the publication database of the WHO's International Agency for Research on Cancer, the Cancer Atlas of the Union for International Cancer Control and the Global Cancer Project Map. A hand search of reference lists of included studies will be conducted. For recency, only articles published over the last 10 years (from 2010 to date) will be considered eligible. No language restrictions will be applied, and any potentially eligible article in a language other than English will be translated using a web-based translation tool. ${ }^{40}$ The preliminary literature search was initiated on 9 November 2020, and completed on 15 January 2021.

The review process will consist of two levels of screening: a title and abstract screening to identify potentially eligible publications and review of full texts to select those to be included in the review based on predefined inclusion/ exclusion criteria. For the first level of screening, two reviewers (CAN and PK) will independently screen the titles and abstracts of all retrieved records from the search output. Articles that are considered relevant by either or both of the reviewers will be included in the full-text assessment. Following the removal of duplicates, full texts of remaining studies will be retrieved. In the second step, the two reviewers will then independently assess the full texts to determine if they meet the inclusion/exclusion criteria. Any discordance in their eligibility assessment will be resolved through consensus between the two researchers. Any further disagreements will be resolved by a third reviewer (JM).

\section{STEP 4: CHARTING THE EVIDENCE}

Two reviewers (CAN and PK) will independently abstract and record all relevant data using a standardised data abstraction tool, adapted from the framework proposed by Carlos and colleagues. ${ }^{41}$ The tool includes four domains: (1) study identification details (article title; journal title; authors; country of the study; language; publication year; host institution of the study); (2) methodological characteristics (study design; study objective or research question or hypothesis; sample characteristics (eg, sample size; sex; age, ethnicity; groups and controls; follow-up duration; validation of measures; statistical analyses); (3) main findings and (4) conclusions. Study eligibility will be reverified at the start of/during data extraction.

Where the relevant outcome data in any article are unclear or missing, the corresponding author will be contacted via email for clarification. Any discrepancies in the abstracted data between the two reviewers will be resolved by discussion, and if a consensus is not reached, a third reviewer (JM) will arbitrate. The first reviewer (CAN) will combine the two spreadsheets of abstracted data for analysis. PK will double-check the entered data for completeness and verify the accuracy of analysis. JM and FMW will review analysed data for accuracy and consistency with protocol.

\section{STEP 5: COLLATING, SUMMARISING AND REPORTING THE EVIDENCE}

Findings of the review will be reported using the Preferred Reporting Items for Systematic reviews and Meta-Analyses extension for Scoping Reviews guidelines. ${ }^{42}$ Findings will be narratively summarised and reported based on themes that will emerge from the charted evidence. Where applicable, quantitative evidence will be aggregated using summary statistics. Diagnostic phases and intervals will be synthesised and reported based on the model of pathways to treatment proposed by Walter, Scott and colleagues which describes five key events in the pathway to care and also identifies the distinct intervals between these phases. ${ }^{1829}$ The model also allows for the assessment of patient-related, healthcare provider and health systemrelated and disease-related factors that could influence the intervals.

As the purpose of a ScR is to aggregate evidence and present a summary of the evidence rather than to evaluate the quality of the individual evidence, this review's overall 
assessment of the strength of the synthesised evidence will be narrative rather than quantitative.

\section{STEP 6: CONSULTATION WITH STAKEHOLDERS}

Stakeholder consultations provide opportunities to enable stakeholders to build on the evidence and offer additional insights, practical meaning and perspectives to the findings of the review. ${ }^{36}$ Hence, we will engage with health policy makers, cancer programme managers, researchers, oncologists and other cancer care providers to help identify grey literature that may not be obtainable from scholarly database searches, as well as providing conceptual and practical insights for guiding the interpretation and dissemination of findings.

\section{PATIENT AND PUBLIC INVOLVEMENT}

As this is a protocol for a ScR of already existing literature, and no participant recruitment will take place, patients were not directly involved in the development of this protocol.

\section{ETHICS AND DISSEMINATION}

This will be a ScR of publicly available literature, with no primary data collection. Hence, it will not require ethical approval. Findings will be disseminated widely through peer-reviewed publication and in various media, for example, conferences, congresses or symposia. This review will provide a user-friendly evidence summary for health policy makers, cancer programme managers and frontline health workers, for informing further efforts at addressing breast and cervical cancer diagnostic delays in LMICs, while identifying opportunities for future research.

\section{Author affiliations}

${ }^{1}$ Cancer Research Initiative, Faculty of Health Sciences, University of Cape Town, Cape Town, Western Cape, South Africa

${ }^{2}$ Women's Health Research Unit, School of Public Health and Family

Medicine,Faculty of Health Sciences, University of Cape Town, Cape Town, Western Cape, South Africa

${ }^{3}$ Department of Public Health, Faculty of Health Sciences, Lira University, Lira, Uganda

${ }^{4}$ Department of Public Health and Primary Care, University of Cambridge,

Cambridge, UK

${ }^{5}$ SAMRC Gynaecology Cancer Research Centre, University of Cape Town, Cape Town, Western Cape, South Africa

\section{Twitter Chukwudi A Nnaji @ChuxNesta_Nnaji}

Acknowledgements The authors wish to thank Namhla Madini of the Faculty of Health Sciences Library, University of Cape Town, for the support and guidance in developing the literature search strategy.

\section{Collaborators Not applicable.}

Contributors JM conceived the study and provided conceptual guidance for the design of the protocol. CAN wrote the first draft of the manuscript. PK supported the development of the Methods section. JM and FMW provided critical insights and guided the coherence of the manuscript. All authors have approved and contributed to the final version of the manuscript.
Funding This research was jointly supported by the Cancer Association of South Africa (CANSA), the University of Cape Town and, the SA Medical Research Council with funds received from the SA National Department of Health, GlaxoSmithKline Africa Non-Communicable Disease Open Lab (via a supporting grant Project Number: 023) and the UK Medical Research Council (via the Newton Fund). Authors retained control of the final content of the publication.

Competing interests None declared.

Patient consent for publication Not required.

Provenance and peer review Not commissioned; externally peer reviewed.

Supplemental material This content has been supplied by the author(s). It has not been vetted by BMJ Publishing Group Limited (BMJ) and may not have been peer-reviewed. Any opinions or recommendations discussed are solely those of the author(s) and are not endorsed by BMJ. BMJ disclaims all liability and responsibility arising from any reliance placed on the content. Where the content includes any translated material, BMJ does not warrant the accuracy and reliability of the translations (including but not limited to local regulations, clinical guidelines, terminology, drug names and drug dosages), and is not responsible for any error and/or omissions arising from translation and adaptation or otherwise.

Open access This is an open access article distributed in accordance with the Creative Commons Attribution Non Commercial (CC BY-NC 4.0) license, which permits others to distribute, remix, adapt, build upon this work non-commercially, and license their derivative works on different terms, provided the original work is properly cited, appropriate credit is given, any changes made indicated, and the use is non-commercial. See: http://creativecommons.org/licenses/by-nc/4.0/.

\section{ORCID IDs}

Chukwudi A Nnaji http://orcid.org/0000-0002-4132-1922

Paul Kuodi http://orcid.org/0000-0003-2483-3499

Fiona M Walter http://orcid.org/0000-0002-7191-6476

Jennifer Moodley http://orcid.org/0000-0002-9398-5202

\section{REFERENCES}

1 Global Burden of Disease Cancer Collaboration, Fitzmaurice C Abate D, et al. Global, regional, and National cancer incidence, mortality, years of life lost, years lived with disability, and DisabilityAdjusted life-years for 29 cancer groups, 1990 to 2017: a systematic analysis for the global burden of disease study. JAMA Oncol 2019;5:1749-68.

2 de Sanjose S, Tsu VD. Prevention of cervical and breast cancer mortality in low- and middle-income countries: a window of opportunity. Int J Womens Health 2019;11:381-6.

3 Demment MM, Peters K, Dykens JA, et al. Developing the evidence base to inform best practice: a scoping study of breast and cervical cancer reviews in low- and middle-income countries. PLoS One 2015;10:e0134618.

4 Bray F, Ferlay J, Soerjomataram I, et al. Global cancer statistics 2018: GLOBOCAN estimates of incidence and mortality worldwide for 36 cancers in 185 countries. CA Cancer J Clin 2018;68:394-424.

5 GLOBOCAN. Cancer today 2018, 2018. Available: http://gco.iarc.fr/ today/home [Accessed 26 Mar 2020].

6 Dalton M, Holzman E, Erwin E, et al. Patient navigation services for cancer care in low-and middle-income countries: a scoping review. PLoS One 2019;14:e0223537-e.

7 Brand NR, Qu LG, Chao A, et al. Delays and barriers to cancer care in low- and middle-income countries: a systematic review. Oncologist 2019;24:e1371-80.

8 Qu LG, Brand NR, Chao A, et al. Interventions addressing barriers to delayed cancer diagnosis in low- and middle-income countries: a systematic review. Oncologist 2020;25:e1382-e1395.

9 Richards MA, Westcombe AM, Love SB, et al. Influence of delay on survival in patients with breast cancer: a systematic review. Lancet 1999;353:1119-26.

10 Emery JD, Shaw K, Williams B, et al. The role of primary care in early detection and follow-up of cancer. Nat Rev Clin Oncol 2014;11:38-48.

11 Neal RD, Tharmanathan P, France B, et al. Is increased time to diagnosis and treatment in symptomatic cancer associated with poorer outcomes? systematic review. Br J Cancer 2015;112 Suppl 1:S92-107.

12 Moodley J, Cairncross L, Naiker T, et al. From symptom discovery to treatment - women's pathways to breast cancer care: a crosssectional study. BMC Cancer 2018;18:312. 
13 Wells KJ, Battaglia TA, Dudley DJ, et al. Patient navigation: state of the art or is it science? Cancer 2008;113:1999-2010.

14 Valaitis RK, Carter N, Lam A, et al. Implementation and maintenance of patient navigation programs linking primary care with communitybased health and social services: a scoping literature review. BMC Health Serv Res 2017;17:116.

15 Jedy-Agba E, McCormack V, Adebamowo C, et al. Stage at diagnosis of breast cancer in sub-Saharan Africa: a systematic review and meta-analysis. Lancet Glob Health 2016;4:e923-35.

16 Tegegne TK, Chojenta C, Loxton D, et al. The impact of geographic access on institutional delivery care use in low and middle-income countries: systematic review and meta-analysis. PLoS One 2018;13:e0203130.

17 Cazap E, Magrath I, Kingham TP, et al. Structural barriers to diagnosis and treatment of cancer in low- and middleincome countries: the urgent need for scaling up. $J$ Clin Oncol 2016;34:14-19.

18 Scott SE, Walter FM, Webster A, et al. The model of pathways to treatment: conceptualization and integration with existing theory. $\mathrm{Br} \mathrm{J}$ Health Psychol 2013;18:45-65.

19 Ginsburg OM, Chowdhury M, Wu W, et al. An mHealth model to increase clinic attendance for breast symptoms in rural Bangladesh: can bridging the digital divide help close the cancer divide? Oncologist 2014;19:177-85.

20 Poum A, Promthet S, Duffy SW, et al. Factors associated with delayed diagnosis of breast cancer in northeast Thailand. $J$ Epidemiol 2014;24:102-8.

21 Grosse Frie K, Samoura H, Diop S, et al. Why do women with breast cancer get diagnosed and treated late in sub-Saharan Africa? perspectives from women and patients in Bamako, Mali. Breast Care 2018:13:39-43.

22 Grosse Frie K, Kamaté B, Traoré CB, et al. Factors associated with time to first healthcare visit, diagnosis and treatment, and their impact on survival among breast cancer patients in Mali. PLoS One 2018;13:e0207928.

23 Tetteh DA, Faulkner SL. Sociocultural factors and breast cancer in sub-Saharan Africa: implications for diagnosis and management. Womens Health 2016;12:147-56.

24 Githaiga JN, Walter FM, Scott SE, et al. Symptom awareness measures for breast and cervical cancer in sub-Saharan Africa: a scoping review. South African Journal of Oncology 2019;3.

25 Mwaka AD, Okello ES, Wabinga $\mathrm{H}$, et al. Symptomatic presentation with cervical cancer in Uganda: a qualitative study assessing the pathways to diagnosis in a low-income country. BMC Womens Health 2015;15.

26 Moodley J, Cairncross L, Naiker T, et al. Understanding pathways to breast cancer diagnosis among women in the Western Cape Province, South Africa: a qualitative study. BMJ Open 2016;6:e009905.
27 Neal RD, Nafees S, Pasterfield D, et al. Patient-Reported measurement of time to diagnosis in cancer: development of the cancer symptom interval measure (C-SIM) and randomised controlled trial of method of delivery. BMC Health Serv Res 2014;14:3.

28 Unger-Saldaña K, Peláez-Ballestas I, Infante-Castañeda C. Development and validation of a questionnaire to assess delay in treatment for breast cancer. BMC Cancer 2012;12:626.

29 Walter F, Webster A, Scott S, et al. The Andersen model of total patient delay: a systematic review of its application in cancer diagnosis. J Health Serv Res Policy 2012;17:110-8.

30 Weller D, Vedsted P, Rubin G, et al. The Aarhus statement: improving design and reporting of studies on early cancer diagnosis. $\mathrm{Br} J$ Cancer 2012:106:1262-7.

31 Battaglia TA, Roloff K, Posner MA, et al. Improving follow-up to abnormal breast cancer screening in an urban population. A patient navigation intervention. Cancer 2007:109:359-67.

32 Drake BF, Tannan S, Anwuri VV, et al. A community-based partnership to successfully implement and maintain a breast health navigation program. J Community Health 2015;40:1216-23.

33 World Health Organization (WHO). Guide to cancer early diagnosis. Geneva, Switzerland: World Health Organization, 2017. http://apps. who.int/iris/bitstream/10665/254500/1/9789241511940-eng.pdf? ua $=1$

34 World Health Organization (WHO). Who report on cancer: setting priorities, investing wisely and providing care for all. Geneva: World Health Organization, 2020.

35 Arksey H, O'Malley L. Scoping studies: towards a methodological framework. Int J Soc Res Methodol 2005;8:19-32.

36 Levac D, Colquhoun H, O'Brien KK. Scoping studies: advancing the methodology. Implement Sci 2010;5:69.

37 Peters MDJ, Godfrey CM, Khalil H, et al. Guidance for conducting systematic scoping reviews. Int J Evid Based Healthc 2015;13:141-6.

38 Fantom N, Serajuddin U. The World Bank's Classification of Countries by Income (English). Policy Research Working Paper no. WPS 7528. Washington, DC: World Bank Group, 2016.

39 McGowan J, Sampson M, Salzwedel DM, et al. PRESS Peer Review of Electronic Search Strategies: 2015 Guideline Statement. J Clin Epidemiol 2016;75:40-6.

40 DocTranslator web site. Available: https://www.onlinedoctranslator. $\mathrm{com} /$ [Accessed 14 Mar 2020].

41 Carlos LL-J, LAPd C, Leopoldo VC. Effectiveness of traditional Chinese acupuncture versus sham acupuncture: a systematic review. Rev Lat Am Enfermagem 2016;24:e2762-e.

42 Tricco AC, Lillie E, Zarin W, et al. PRISMA extension for scoping reviews (PRISMA-ScR): checklist and explanation. Ann Intern Med 2018;169:467-73. 